\title{
Clinical and Instrumental Objective Evidence of the Efficacy of a New Water-Based Nail- Strengthening Solution Containing Pistacia lentiscus and Hyaluronic Acid Applied for Up to 6 Months to Improve the Appearance of Weak, Brittle Nails
}

\author{
Bianca Maria Piraccini · Corinne Granger - Aurora Alessandrini · Nicolò Brandi • \\ Francesca Bruni · Victor Desmond Mandel - Giovanni Pellacani · Michela Starace
}

Received: September 18, 2019 / Published online: November 20, 2019

(C) The Author(s) 2019

\section{ABSTRACT}

Introduction: Brittle nails are fragile or split nails; they affect $20 \%$ of the population and may be primary or secondary to different conditions. The aim of our studies was to evaluate the efficacy and tolerability of a new waterbased nail-strengthening treatment containing hyaluronic acid and Pistacia lentiscus with daily application for a period of 1-3 months for one study $(n=30)$ and up to 6 months for a second study $(n=30)$.

Methods: In total, we enrolled 60 patients of both sexes with brittle and weak nails due to primary or secondary causes and evaluated the

Enhanced Digital Features To view enhanced digital features for this article go to https://doi.org/10.6084/ m9.figshare.10101413.

B. M. Piraccini $(\varangle) \cdot$ A. Alessandrini · N. Brandi · F. Bruni · M. Starace

Department of Experimental, Diagnostic and Specialty Medicine-Division of Dermatology, University of Bologna, Bologna, Italy

e-mail: biancamaria.piraccini@unibo.it

C. Granger

Innovation and Development ISDIN, Barcelona, Spain

V. D. Mandel · G. Pellacani

Dermatology Unit, Surgical, Medical and Dental Department of Morphological Sciences Related to Transplant, Oncology and Regenerative Medicine, University of Modena and Reggio Emilia, Modena, Italy efficacy of this new product using subjective and objective methods: clinical evaluation, assessment of photographs, onychoscopy evaluation, investigator and patient global assessment, dynamic optical coherence tomography (D-OCT) and reflectance confocal microscopy (RCM).

Results: Studies subjects presented a statistically significant improvement in global assessment scale (GAS) scores at 14 days $(\mathrm{GAS}=1.7 \pm 0.6), 1$ month $(\mathrm{GAS}=1.4 \pm 0.7)$ and at 3 months (GAS $=1 \pm 0.7$ ) versus the GAS score obtained before treatment $(1.9 \pm 0.5)$ $(p<0.0001)$. From the Italian study at 6 months $(n=30) 76 \%$ of the patients had an improvement in their nail appearance. Reduction in nail plate roughness with improved nail resistance and decreased distal breakage were the most evident benefits, demonstrated on clinical and instrumental evaluations. No side effects were reported. All patients reported an improvement in nail appearance after using the product for 1 month, 3 months and 6 months, and had a positive opinion on the product.

Conclusions: This new product is an effective, safe, and easy-to-use option for topical treatment of brittle nails and primary nail fragility and an adjuvant therapy in secondary nail fragility. Moreover, its ease of application and cosmetic qualities allow good compliance.

Funding: ISDIN. 
Keywords: Brittle nails; Nails; OCT; Onychoscopy; RCM; Weak nails

\section{Key Summary Points}

\section{Why Carry Out This Study?}

Brittle nails affect $20 \%$ of the population, with women affected more often than men.

Nail fragility can present an important cosmetic problem, which may also interfere with everyday activities.

Treatment of brittle nails is usually a big challenge for dermatologists, due to the slow growth rate of the nail plate and the difficulty in getting actives to penetrate the nail tissue. Thus, the ideal topical product for nail fragility should be easy to apply and cosmetically acceptable.

\section{What Was Learned from the Study?}

The conclusions of our studies indicate that this new product is an effective, safe, and easy-to-use option for topical treatment of nail brittleness, and its cosmetic qualities facilitate better treatment compliance.

The modern and sophisticated techniques used in these clinical trials allow us to demonstrate the true efficacy of this topical product in improving nail resistance and thickness, and hydrating and protecting the periungual skin.

\section{INTRODUCTION}

Brittle nails are characterized by nail fragility and splitting, with a rough, opaque nail plate. This condition affects $20 \%$ of the population, with women affected more often than men [1]. Environmental and occupational factors, such as increasing age, can lead to progressive dehydration of the nail plate and play an important role in the development of nail brittleness (idiopathic/primary nail fragility) [2, 3]. Nail exposure to exogenous irritants and allergens also contributes to fragility, damaging the periungual tissues and impairing their protective role. The cuticle is a horny expansion of the proximal nail fold that seals it to the nail plate, protecting the underlying matrix, and its removal can worsen nail fragility. Causes of secondary nail fragility include systemic drugs, such as retinoids and cancer chemotherapeutic agents, systemic diseases, such as severe iron deficiency, and traumatic and dermatological nail diseases. These conditions may impair nail matrix activity, leading to the growth of a weak, fragile nail plate, or may mechanically damage the existing nail plate.

Nail fragility can present an important cosmetic problem, which may also interfere with everyday activities [4]. Patients usually complain that their nails are dry, weak, easily breakable and unable to grow long $[4,5]$. The nail plate surface loses its shine and smoothness.

Treatment of brittle nails is usually a big challenge for dermatologists, due to the slow growth rate of the nail plate and the difficulty in getting actives to penetrate the nail tissue. One of the main goals for successful topical therapy is the cosmetic acceptability of the product, which must be applied for several months to obtain clear results. Thus, the ideal topical product for nail fragility should be easy to apply, with no sticky texture or unpleasant odor, and should not leave an appearance of "varnished" nails: these characteristics will improve adherence to treatment in male and female patients and, in turn, improve the efficacy.

The aim of our studies was to evaluate the efficacy, tolerability and patient compliance of a new topical nail-strengthening treatment containing cationic hyaluronic acid and mastic oil (Pistacia lentiscus).

\section{METHODS}

A preliminary 1-month study, subsequently extended to 3 months, in Portugal, and a 
second 6-month study in Italy, included volunteers with primary and secondary weak and brittle nails. Globally, a cohort of 60 patients was initially included in the study and evaluated at 1 month. Seven subjects from the Portuguese cohort were not invited to continue the study after the first visit and 1 subject withdrew before completing the 3-month study. Therefore, 52 subjects were evaluated at 6 months. For all patients, the diagnosis was established on clinical grounds and from their medical history. When suspected, onychomycosis was excluded through clinical examination, direct microscopy and/or culture. None of the patients were receiving systemic or other topical treatments.

\section{Study Populations}

Portuguese study: 30 female patients (mean age 46.28 , from 23 to 63 years) with primary fingernail fragility were evaluated for 1 month, of which 23 continued to follow-up at 3 months of treatment; however, one subject was lost to follow-up at 3 months. Therefore, 22 subjects were evaluated at 3 months.

Italian study: 30 patients, 9 males and 21 females (mean age 52.37, from 18 to 82 years). In three patients nail fragility was due to trachyonychia associated with alopecia areata, in two patients it was due to onychotillomania and in three patients it was due to mild nail lichen planus confirmed by histopathology. In one patient nail fragility was a result of chronic paronychia. The other 21 patients were diagnosed as having idiopathic nail fragility, involving the fingernails in 14 patients and the toenails in seven patients.

\section{Product Evaluated}

The product tested is a new water-based nailstrengthening solution specially formulated with a combination of active ingredients (silanediol salicylate and Pistacia lentiscus gum) that increase the quantity and quality of silicon and keratin in the nails. The gum from the tree Pistacia lenticus contains numerous biologically active molecules including essential oils of which $\alpha$-pinene, b-myrcene, b-pinene, linalool, limonene and caryophyllen are the main constituents. The polymer polymyrene represents about $25 \%$ of the gum. The biologically active substances are in part triterpenic acids (C29-C31), including masticadienonic acid, isomasticadienonic, oleanonic acid and moronic acid. Pistacia lentiscus gum has demonstrated in vitro that it increased the synthesis of hard keratins (K31, K83, K85) and in vivo it improved nail strength and thickness (data on file). The product also contains cationic hyaluronic acid that adheres to the surface and deeply moisturizes the nails and cuticles. This combination of ingredients (patent pending) helps the natural process of nail repair and growth. The product was applied to the entire nail surface and the periungual tissues once daily for 3 months (Portugal) or 6 months (Italy).

\section{Clinical Parameters}

In both studies, the evaluation of treatment efficacy and tolerability was performed after 2 weeks (V2), 1 month (V3), and 3 months (V4); in the Italian study an additional 6-month evaluation (V5) was performed (Figs. 1, Fig. 2). Both studies evaluated the fragility of target nails according to the investigator's assessment using a 4-point global assessment scale (GAS): 0 (clear of any sign of splitting, ridges or grooves; normal nail plate thickness), 1 (mild tendency to breakage; very few distal horizontal splits and longitudinal grooves), 2 (moderate tendency to breakage with horizontal splitting of the nail; few deep ridges and longitudinal grooves; distal longitudinal fissures of the nail plate free edge; mild thinning of the nail) and 3 (severe lamellar or transversal splitting; more than $70 \%$ of the nail showing deep ridges and grooves; extended longitudinal fissures of the nail plate margin; a dramatic reduction of the nail plate thickness).

In addition, in both studies, patient assessment scales (PAS) of treatment efficacy were performed with a specific questionnaire with a scale from 0 to 3 , where 0 indicated no improvement; 1 , some improvement; 2 , clear improvement; and 3 , very good improvement. 


\section{Portugal Study}

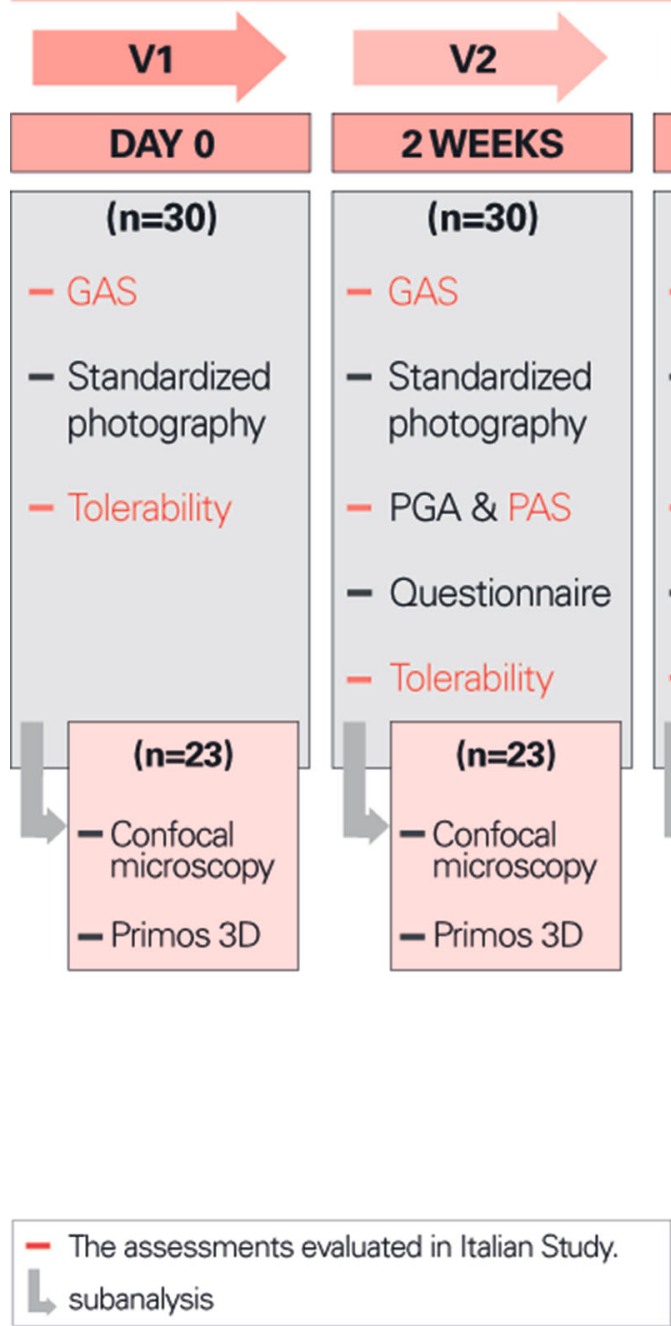

Fig. 1 Portuguese study design. Assessments in 30 subjects: global assessment scale (GAS), standardized photographs, physician global assessment (PGA), patient assessment scale (PAS), questionnaire and tolerability. A

For both studies, each follow-up visit (week 2, months 1, 3 and 6) included standardized photos of the target nails, expert investigator grading and subject-reported outcome measures.

During and at the end of treatment, patients were asked to give their opinion about the efficacy and the cosmetic properties of the product (e.g., ease of application).

Finally, the tolerability of the product was evaluated through examination of

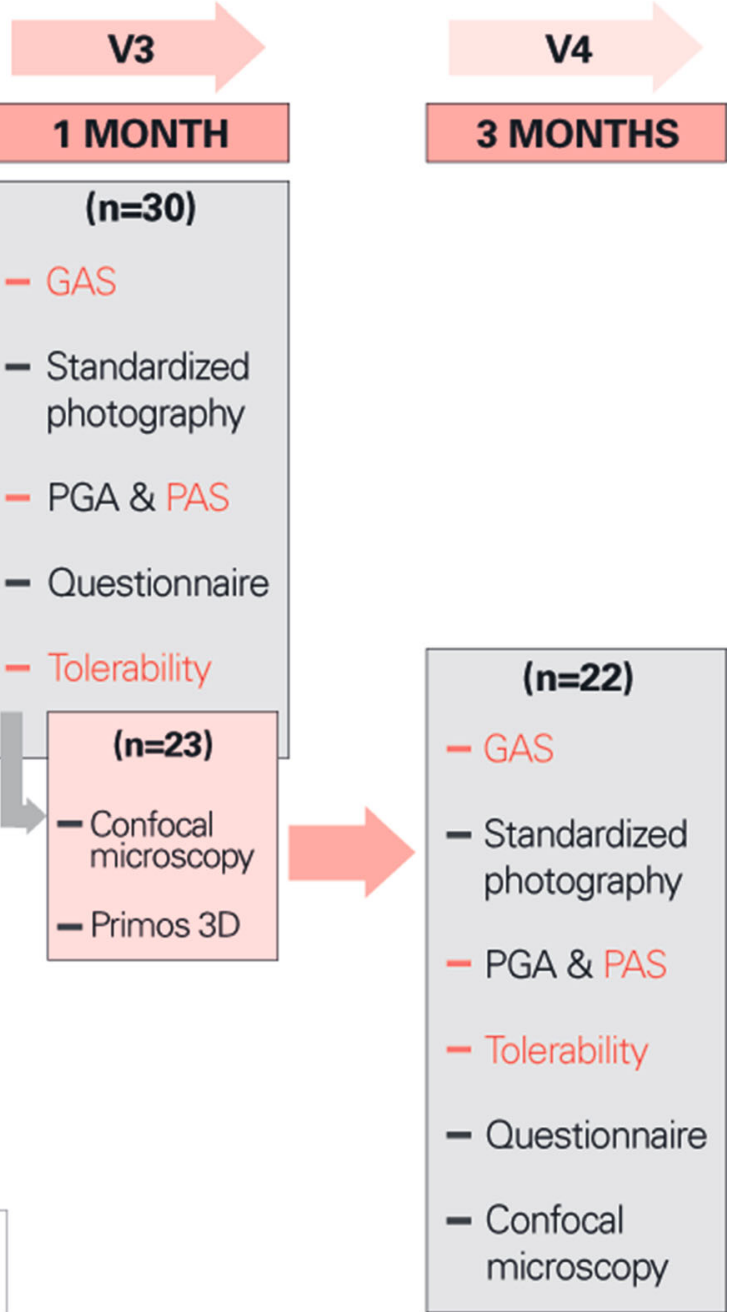

sub-analysis of 23 of the subjects was performed with confocal microscopy, Primos 3D, and standardized photographs. With one drop-out, the study was extended for 22 subjects to 3 months

investigational areas (nails and skin around the nails) and evaluation of other symptoms (pain, stinging/burning) manifested by subjects at each visit.

\section{Evaluation Techniques}

\section{Confocal Microscopy}

A Vivascope 1500 (Mavig GmbH, Munich, Germany) in vivo confocal microscopy system was used to obtain uniform imaging of the 


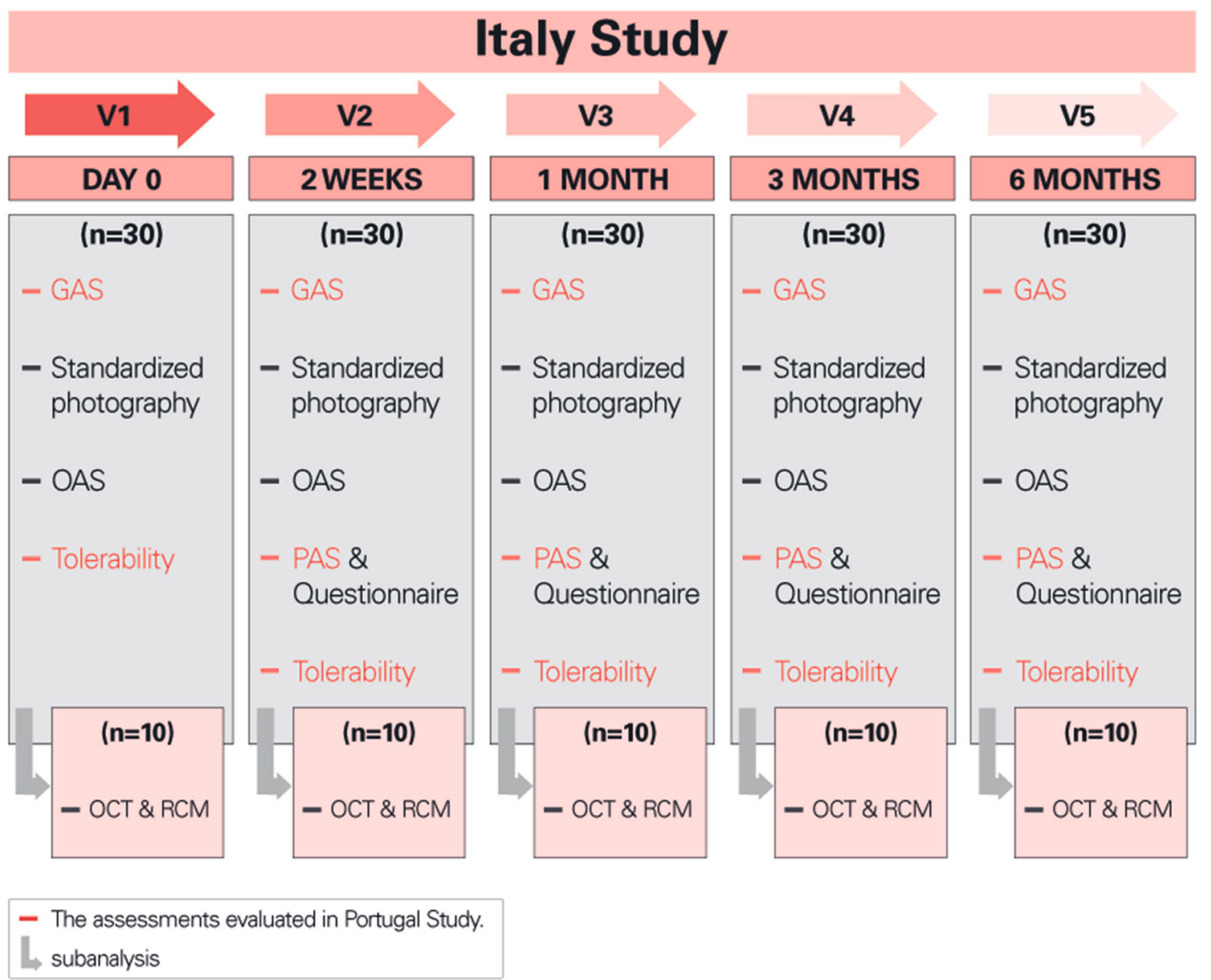

Fig. 2 Italian study design. Assessment in 30 subjects: global assessment scale (GAS), onychoscopy assessment scale (OAS) and patient assessment scale (PAS) performed

nails. From these 2-dimensional images, the total nail thickness, thickness of the superficial layer of the nail, and the density of the nail were assessed [6, 7].

\section{PRIMOS 3D}

PRIMOS (phaseshift rapid in vivo measurement of skin) optical 3D is an in vivo optical measurement device that uses a fringe projection technique based on micromirror display devices. A fringe standard is projected on the nail and detected by the system's CCD camera. The $3 \mathrm{D}$ effect is calculated from the deflection in the fringes which provides quantitative information on the nail profile $[8,9]$.

The standard roughness was calculated from the full aligned image. The parameters at all follow-up visits. A sub-analysis of 10 of the subjects was performed with reflectance confocal microscopy (RCM) and optical coherence tomography (OCT)

evaluated were $\mathrm{Ra}$ (arithmetic mean of nail surface) and Rz (mean of the 5 highest "peaks" and 5 lowest "valleys" in the image area).

\section{Onychoscopy}

Onychoscopy evaluation was supported and documented by standardized dry videodermoscopy (Fotofinder Thricoscan ${ }^{\circledR}$ ) of the target nail. The evaluation used a 4-point onychoscopy assessment scale (OAS), from 0 (clear of any sign of splitting, ridging or grooves) to +3 (severe lamellar o transversal splitting, more than $70 \%$ of the nail showing deep ridging and grooves; extended longitudinal fissures of the nail plate margin). 


\section{Dynamic Optical Coherence Tomography (D- OCT)}

D-OCT was performed on the top of the nail plate through the proximal nail fold and from the end of the nail plate through the distal margin of the considered nail, by means of a commercially available D-OCT (VivoSight $^{\circledR}$ : Michelson Diagnostics Ltd, Maidstone, UK) and 3D image reconstruction software. The VivoSight ${ }^{\circledR}$ OCT scanner uses eye-safe infrared laser light to obtain a 3D block of image data at a high resolution $(<10 \mu \mathrm{m})$. OCT has a central wavelength of $1305 \mathrm{~nm}$ and scans an area of $6 \mathrm{~mm}$ by $6 \mathrm{~mm}$ up to a depth of $2 \mathrm{~mm}$. D-OCT allows the description of blood flow in vivo and visualization of the tissue microvasculature. In order to minimize normal anatomical variation, the same depth was chosen for all images using the software tool "OCTFITTER" (University of Modena and Reggio Emilia, Modena, Italy) that provide horizontal D-OCT images at three selected depths $(150,300$ and $500 \mu \mathrm{m})$ [10-12].

\section{Reflectance Confocal Microscopy (RCM)}

RCM (VivaScope ${ }^{\circledR}$ 3000: Mavig GmbH, Munich, Germany) was used to visualize the nail plate. It uses a diode laser at $830 \mathrm{~nm}$ and $<22 \mathrm{~mW}$ power at the tissue level, for the in vivo imaging of nails with cellular resolution. Each individual RCM image corresponds to a horizontal section of approximately $1 \times 1 \mathrm{~mm}$, at a selected tissue depth, with a lateral resolution of $1 \mu \mathrm{m}$. Crodamol oil was employed as a coupling medium between the probe and the nail surface. The images were taken from the top of the nail plate through the nail bed and at the distal margin of the nail. RCM and D-OCT (cross-sectional and en face) images were obtained from each patient before, during, and after the application of the nail gel for qualitative visual comparison [13]. Reflectance confocal microscopy (RCM) (VivaScope $^{\circledR} 1500$ ) assessed the total nail thickness, the thickness of the superficial layer of the nail and the density of the nail. The parameters were measured according to a routine that included a two-dimensional evaluation of the image of the nail obtained. The results were presented in pixels.

\section{Ethical Consideration}

Due to the cosmetic nature of the intended use of the product studied, ethics committee approval was not required. Both studies were performed in line with the Declaration of Helsinki (1964) and its subsequent amendments. In both studies, the subjects were informed about the expected benefits of the tested products and the constraints of study participation and signed an informed consent form, including consent for the use of photographs taken during the studies, prior to the start of the studies.

\section{Statistical Analyses}

Portuguese study: values are expressed as the mean and standard deviation (SD). A Willcoxon's test for paired samples was performed to evaluate changes between the data obtained at baseline and at each time-point. Differences were considered significant at $p<0.05$. Statistical analysis was performed using SPSS software (version 20.0; SPSS Inc, Chicago, IL, USA).

Pool analysis: the analysis has been carried out on the set of data from the different individual databases of each of the aggregate studies, taking into account that exactly the same information has been collected with the same schedule. Continuous variables have been described by the number of valid cases, mean and standard deviation. Comparisons of categorical variables have been made using the chisquare test. In the case of continuous variables, the Student $t$-test was used for independent data. For all comparisons a level of statistical significance of 0.05 bilateral was considered. All analyses were performed on the data set using all available information with intention to treat (ITT) criteria. Statistical analysis was performed using the SAS (Statistical Analysis System) program, version 9.2.

\section{RESULTS}

In total, 60 subjects were included in the two studies. In the Portuguese study, 30 subjects enrolled for 1 month of treatment and 23 
females continued to the extension part of the study to 3 months of treatment; however, one subject was lost to follow-up at 3 months. Therefore, 22 subjects were evaluated at 3 months.

In the Italian study, 30 patients were enrolled and completed the 6-month study.

Three-month data were available for 52 patients and 6-month data for 30 subjects.

No treatment-related side effects were recorded.

\section{Clinical Evaluation via Global Assessment Scale (GAS) for Both Studies}

In both studies the same evaluated parameters have been considered at the same time points. So the data have been presented individually and pooled for these parameters.

In the Portuguese study the subjects presented a statistically significant improvement in GAS scores at 14 days $(\mathrm{GAS}=1.6 \pm 0.5)$, 1 month $(\mathrm{GAS}=1.2 \pm 0.5)$ and 3 months $(\mathrm{GAS}=1.1 \pm 0.4) \quad$ versus the GAS score obtained before treatment $(1.8 \pm 0.5)$ $(p<0.05)$. At 1 month of treatment, the percentage decrease in GAS and, therefore, improvement in nail condition, was $31.8 \%$ versus D0. Moreover, at 1 month and 3 months of treatment, $3 \%$ and $4.5 \%$ of subjects, respectively, obtained complete resolution with a GAS $=0$.

In the Italian study, at the end of the 6 months of treatment, 17 of 30 patients
(56.67\%) obtained considerable improvement, the majority associated with complete resolution of the nail plate abnormalities $(\mathrm{GAS}=0)$ (Fig. 3); improvement was noted both in fingernails and toenails (Fig. 4). Six patients (20\%) showed a mild improvement $(\mathrm{GAS}=1)$ while the remaining seven patients $(23.3 \%)$ remained stable $(G A S=2)$. In total, $76 \%$ of the patients showed an improvement in their nail appearance. When considering patients with secondary nail fragility, the two patients with onychotillomania had total resolution of nail plate abnormalities, despite the presence of periungual blood crusts demonstrating the persistence of the habit tic. A completely healthy nail was seen in the patient affected by chronic paronychia at the 6-month visit, with improvement of the nail plate and of the periungual tissues and regression of the proximal nail fold inflammatory signs (Fig. 5). Marked improvement of nail plate resistance with a decrease in longitudinal fissures was also seen in the three patients with mild nail lichen planus.

With all the study data pooled together, the subjects presented statistically significant improvement in GAS scores at 14 days (GAS = $1.7 \pm 0.6), \quad 1$ month $\quad($ GAS $=1.4 \pm 0.7)$ and 3 months (GAS $=1 \pm 0.7$ ) versus the GAS score obtained before treatment $(1.9 \pm 0.5)$ $(p<0.0001)$. In total, 52\% $(n=60)$ and $86 \%$ $(n=52)$ of the patients showed a decrease in the GAS assessment score and, therefore, an improvement in their nail appearance at 1 month and 3 months, respectively.

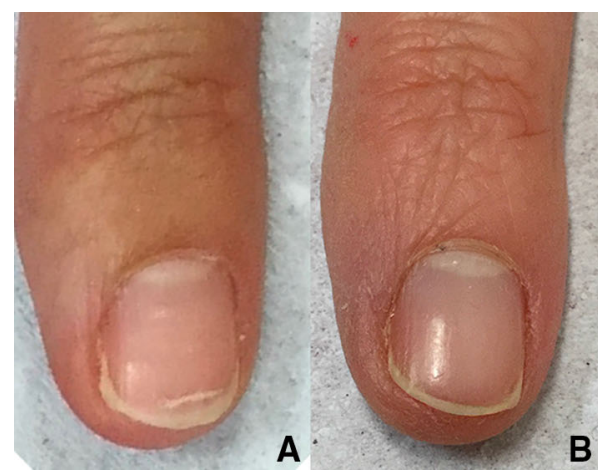

Fig. 3 Complete resolution of idiopathic fingernail fragility and distal splitting after 6 months of treatment: clinical examination shows a smooth shiny nail plate with

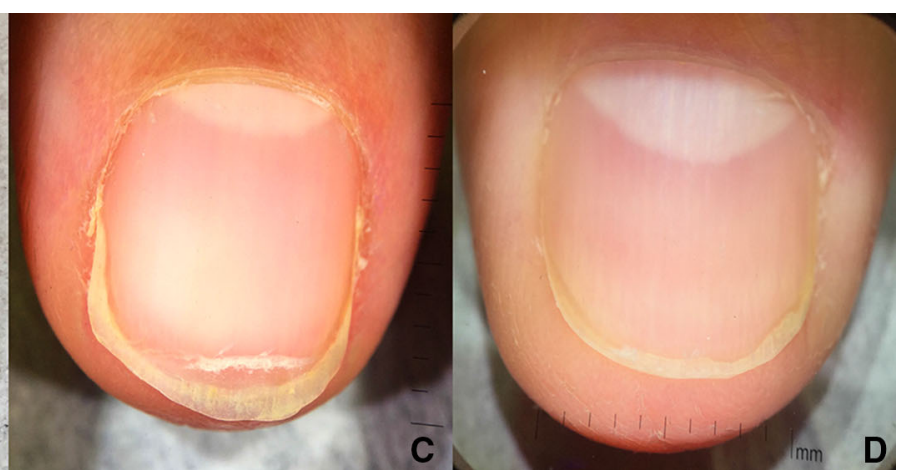

regular free edge (a, b); onychoscopy shows a regular nail plate surface and hydrated healthy cuticle and periungual skin $(\mathbf{c}, \mathbf{d})$ 

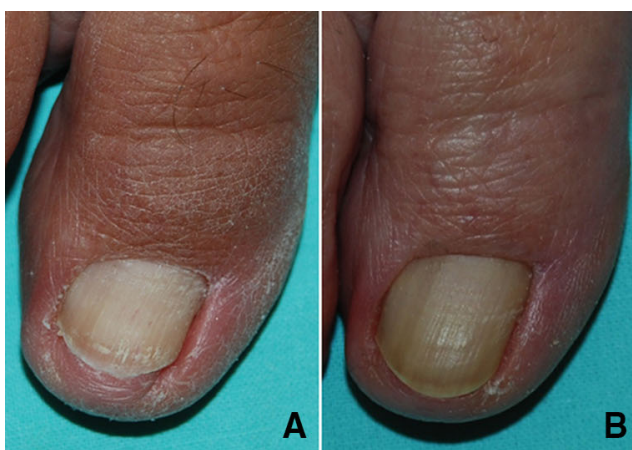

B
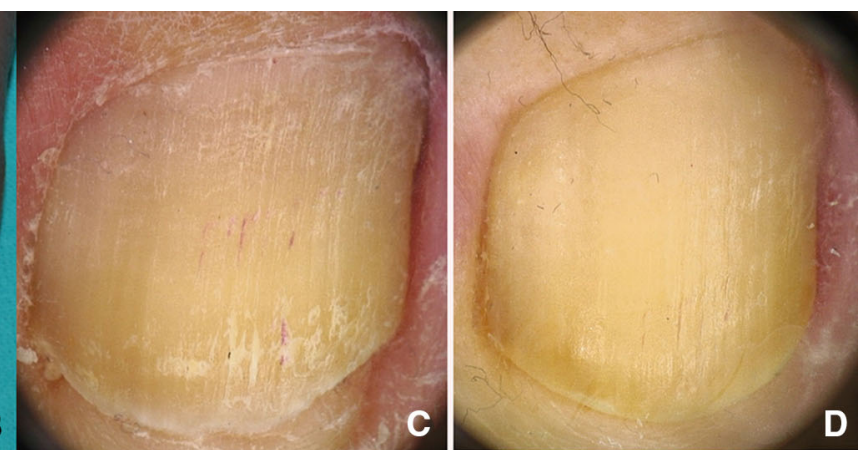

(a, b); onychoscopy shows a smooth nail plate surface with regular distal margin and hydrated healthy cuticle and periungual skin $(\mathbf{c}, \mathbf{d})$
Fig. 4 Complete resolution of idiopathic toenail fragility after 6 months of treatment: clinical examination shows disappearance of longitudinal grooves and superficial scales
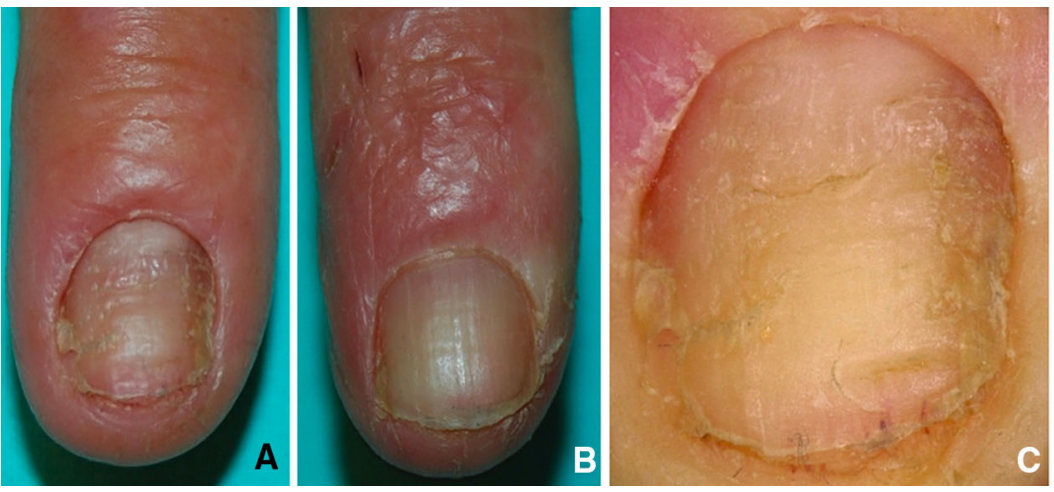

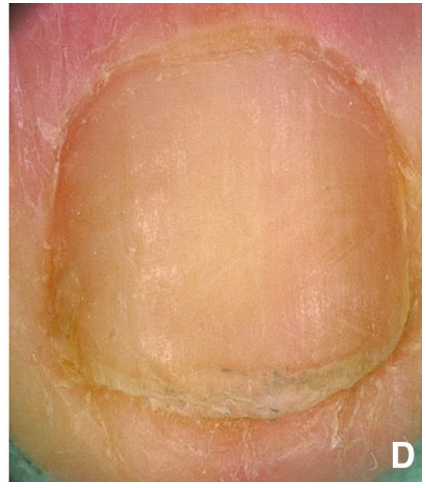

onychoscopy shows a regular nail plate surface and decreased scaling and inflammation of the periungual tissues $(\mathbf{c}, \mathbf{d})$

very good or clear improvement and 12/52 (23\%) reported some improvement; none reported "no improvement".

In the Portuguese study, all patients also defined the product as easy to apply, with a pleasant texture and cosmetic qualities, as it did not leave sticky residues on the nail and periungual tissues: overall, $95.2 \%$ of the subjects had a positive general opinion of the product. All patients reported that their nails were smoother, more hydrated, with an improved appearance including the cuticles (see Table 1). In the Italian study, all patients reported that the product was easy to apply and quickly absorbed without leaving residues in the applied areas. In fact, almost all of the patients had excellent compliance and defined the 
Table 1 Portuguese study: users' responses on the efficacy and cosmetic qualities of the product

\begin{tabular}{ll}
\hline Question & \% Subjects in agreement \\
\hline The product hardens the nails & 93.3 \\
The product leaves nails hydrated & 93.3 \\
The product helps stop nails breaking & 86.7 \\
The nail surface is smoother & 83.3 \\
The overall appearance of the nails is improved & 93.3 \\
After application nails feel cared for & 96.7 \\
After application nails feel comfortable & 96.7 \\
Nails are more beautiful & 90.0 \\
The appearance of the cuticles is improved & 73.3 \\
Nails grow faster than before & 76.7 \\
Nails feel protected & 86.7 \\
The nails now have a more natural color & 80.0 \\
The product is easy to apply & 100 \\
The product spreads easily on the nail & 100 \\
The product has a pleasant fragrance & 86.7 \\
The product has a pleasant texture & 100 \\
The product does not leave a sticky sensation on the nails & 93.3 \\
The product does not leave sticky residues on the nails & 93.3 \\
The product packaging is easy to use & 90.0 \\
I have never seen packaging for a nail product like this & 90.0 \\
This packaging makes the application of the product more reliable & 96.7 \\
\hline
\end{tabular}

product as aesthetically pleasing and effective with improvement evident from the beginning.

\section{Instrumental Evaluations}

Onychoscopy assessment at the end of the 6-month study revealed that signs of splitting, ridges and grooves had completely disappeared in 16 patients $(53.33 \%$ OAS $=0$ ) (Figs. 3,4$) ; 10$ patients $(33.33 \%)$ still showed very few distal signs of nail fragility (OAS +1 ) (Fig. 5) and only 4 patients (13.33\%) still had a few deep ridges and longitudinal grooves (OAS +2 ). Observation of the periungual skin showed complete disappearance of scaling with normally hydrated skin and a healthy cuticle in all cases (Fig. 4).

Confocal microscopy showed a statistically significant increase in thickness of the superficial layer in the subjects' most representative nail at 1 month and 3 months, representing an increase of $12.8 \%$ at 1 month versus initial data.

Nail density was also evaluated in the study, showing a significant statistical increase in nail density of $20.4 \%$ at 1 month and $39.8 \%$ at 3 months versus initial data.

PRIMOS 3D evaluation showed a statistically significant decrease in $\mathrm{Ra}$ - that is, an antiroughness effect - after 1 and 3 months. The mean roughness (Ra) decreased 5.8\%, 8.1\% and 

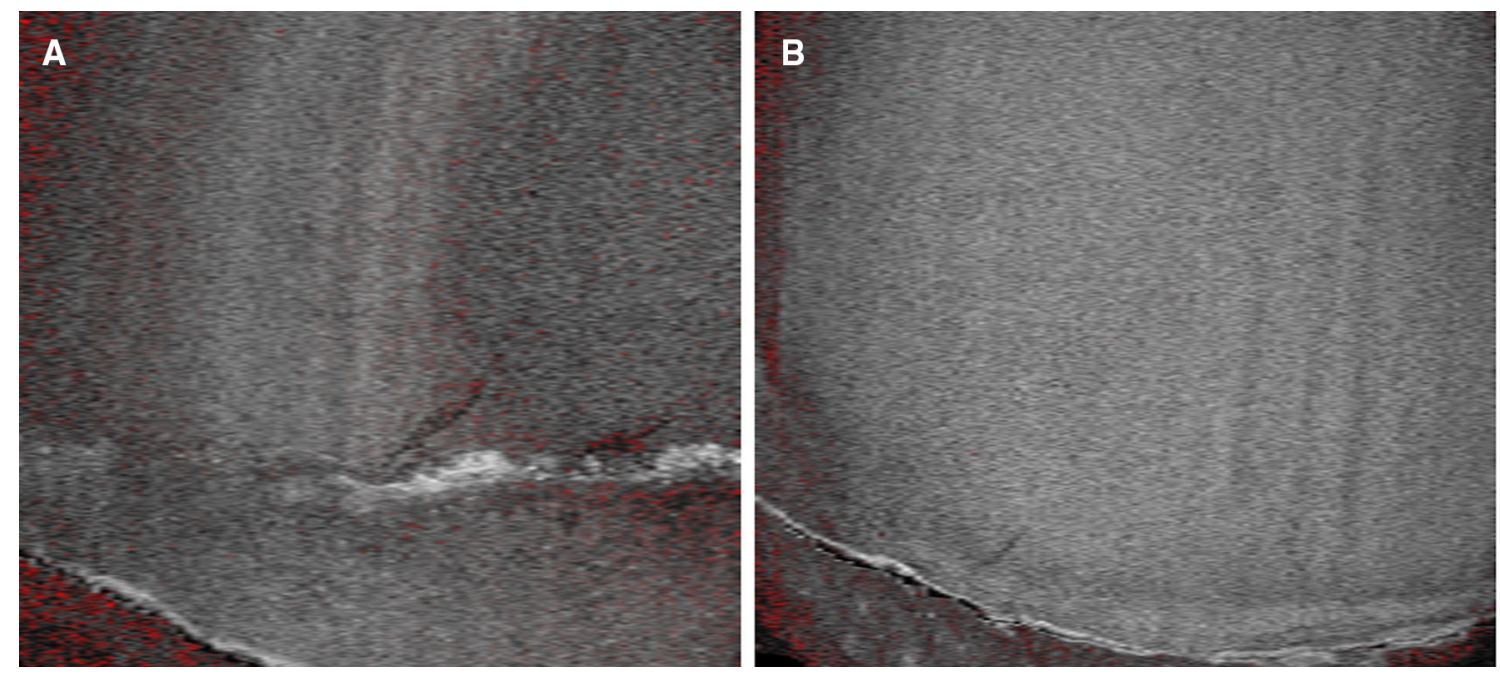

Fig. 6 D-OCT of the distal nail of the patient from Fig. 1, (a) before and after 6 months of treatment: the nail plate surface is more regular and hydrated $(\mathbf{b})$

$17.1 \%$ versus baseline after 14 days, 1 month and 3 months of product application, respectively. The evaluation of the $\mathrm{Rz}$ parameter showed a similar trend.

D-OCT evaluation before treatment (V1) showed a nail plate characterized by an irregular, wavy aspect with a rough surface in all the patients evaluated. Longitudinal ridges were also observed and appeared as multiple hyporeflective grayish to black full-thickness grooves, parallel to each other, resembling the "string of beads" sign on dermoscopy. These OCT signs were observed on the top of the proximal and medial nail plate, while delamination and breakage of the nail plate were visible on the free margin. All these OCT features were milder in seven patients and more pronounced in three patients. At the end of the study, in all patients the nail appeared harder and thicker and the aspect of the nail plate was more regular and less wavy, with a reduction in the hyporeflective grayish grooves. Longitudinal ridges and distal splits were still observed only in 3 severe cases, but they were fewer and less deep. The improvement was evident at both the proximal and distal part of the nail plate (Fig. 6). Measurement of nail thickness before treatment and after 3 months revealed a statistically significant increase in the thickness of the superficial layers of the nail plate $(+7.5 \%)$, the part that undergoes distal splitting and ridging when the nails are brittle. Nail density also increased during the treatment period, with a $20.4 \%$ increase and a $38.9 \%$ increase after 1 and 3 months, respectively. D-OCT evaluation of nail roughness showed decreased nail roughness evident after only 2 weeks of treatment $(-5.5 \%)$ and a $19.2 \%$ decrease in nail roughness at the 3-month follow-up visit.

RCM evaluation before treatment (V1) revealed a homogeneous presence of canaliform ridges on the top of the nail plate with bright white pin-point structures with a homogeneous distribution at the free margin of the nail. At the end of the study (V5), RCM revealed a clear reduction in the canaliform ridges and bright white pin-point structures, especially in the less severe patients. The nail plate appeared brighter and more regular.

\section{DISCUSSION}

Treatment of brittle nails is often a challenge for the dermatologist, for two main reasons: (1) it is difficult for the actives to penetrate the nail plate and particularly to penetrate simultaneously both the nail plate and periungual tissues. The nail plate acts as a hydrophilic membrane, so topical ointments, creams and greasy products cannot penetrate it and have no 
therapeutic effect [14]. The opposite is true of the periungual skin, which should also be a target of therapy, as the health of the nail plate is a result of the normal function of the nail matrix and the protective role of the proximal and lateral nail folds. Most of the available treatments only target the nail plate, as they are generally some form of hydrophilic lacquer or gel that deposit a coating on the nail plate. (2) The nail has a very slow growth rate and topical therapies need several months of use before showing any efficacy. If a product does not have good cosmetic qualities and is difficult to apply, compliance with treatment stops quickly, before seeing any results.

The water-soluble nail strengthener containing Pistacia lentiscus and hyaluronic acid tested in this study is specifically designed to be both effective and easy to use. The gel comes out from a "pen" with a simple click and it is easy to dose: 1 click per nail. Application is quick and easy: the brush is run over the periungual tissues and the nail plate, easily spreading a transparent gel with a pleasant texture that does not leave a sticky sensation or residue on the nails. Compared with other products on the market for the treatment of nail fragility, the new gel is more convenient: it is easy to carry and apply, and combines treatment for the nail plate and for the cuticle and periungual tissues. Of note, $90 \%$ of the patients who completed the study, including males, would buy the product and 95\% would recommend the product to others.

The innovative combination of ingredients (patent pending) of this gel formulation explains its quick and marked efficacy:

1. Hyaluronic acid boosts hydration capacity, a fundamental requirement since brittle nails are a sign of dehydration and appear when the amount of water in the nails is reduced to less than $16 \%[15,16]$. The new patent-pending formulation of hyaluronic acid contained in the water-soluble nail strengthener has a very high adhesive capacity, due to its positive charges, which makes it possible for the product to stay longer on the skin and nails. This characteristic is particularly important when dealing with dryness of the nails. Moreover, as the product is spread on the cuticle and periungual skin, it acts as a moisturizing agent in this sensitive area of the nail apparatus. The periungual tissues are an important component of the nail apparatus, acting as protective barriers against penetration of irritants and allergens into the nail matrix (cuticle) and the lateral nail plate margins (lateral folds): their integrity is therefore a prerequisite for a healthy resistant nail plate. The periungual skin can be damaged in several exogenous and endogenous nail conditions, e.g., chronic paronychia, hand contact dermatitis, psoriasis and mechanical nail dystrophies. An effective treatment for nail fragility should therefore target both the nail plate and the periungual skin, providing hydration and restoring its normal keratinization.

2. Mastic oil is an aromatic vegetable resin produced by the plant Pistacia lentiscus. Its active components stimulate the synthesis of hard keratins and keratin-associated proteins, increasing nail thickness and strength. It has been demonstrated in vitro that it increased the synthesis of hard keratins (K31, K83, K85) and in vivo it improves nail strength and thickness (data on file).

3. Exogenous silicium supports nail growth and improves the nail appearance. Silicium is also a normal component of the skin and its addition to the periungual tissues reinforces their normal growth.

Out of the clinical results, an in vitro study has been performed with the water-soluble gel nail strengthener, using a well-defined bovine hoof membrane model [17] to evaluate the nail treatment efficacy. After 14 days of application, the tested product showed a statistical improvement in firmness and surface characteristics of the nails at the same level as a reference product Betralfatrus, while no improvement was found with vehicle alone (data on file).

Another innovation of this study lies in the methods used to evaluate nail brittleness before and after treatment. Besides investigator and 
patient evaluation, which are important in any trial, and reflect the overall effectiveness and tolerability of a product, we also used highly sophisticated, objective techniques: full nail photographs, onychoscopy, dynamic optical coherence tomography (D-OCT), and reflectance confocal microscopy (RCM).

Onychoscopy is one of the best tools for evaluating nail fragility and tendency to break, as it allows observation at high magnification of the nail surface: a normal nail plate is shiny and smooth, without longitudinal or transverse creases and grooves; the distal margin is regular and not split [18]. In contrast, brittle nails show longitudinal and transverse ridges and grooves, lamellar splitting and longitudinal fissures.

D-OCT and RCM have only recently been applied to the study of nail dystrophies [19-21]. They are expensive and sophisticated techniques that allow for the first time the measurement of different nail parameters in vivo: in our study, by using D-OCT and RCM we were able to measure nail thickness, density and roughness, providing a new and objective evaluation of nail fragility treatment.

In both studies the results were consistent when using the same clinical scales for evaluation.

One limitation of the studies is their openlabel design; however, the objective criteria used, such as onychoscopy, PRIMOS 3D, confocal microscopy, OCT, and RCM support the results obtained.

\section{CONCLUSIONS}

In conclusion, the results obtained in both clinical studies indicate that this new product containing hyaluronic acid and Pistacia lentiscus is an effective, safe, and easy-to-use option for topical treatment of nail brittleness and tendency to breakage. Moreover, its ease of application and cosmetic qualities facilitate better treatment compliance. The modern and sophisticated techniques used in these clinical trials allow us to demonstrate a true efficacy of this topical product in improving nail resistance and thickness and hydrating and protecting the periungual skin.

\section{ACKNOWLEDGEMENTS}

We thank the participants of the study.

Funding. This study and the Rapid Service Fee were funded by ISDIN.

Authorship. All authors had full access to all of the data in this study and take complete responsibility for the integrity of the data and accuracy of the data analysis. All named authors meet the International Committee of Medical Journal Editors (ICMJE) criteria for authorship for this article, take responsibility for the integrity of the work as a whole, and have given their approval for this version to be published.

Disclosures. Corinne Granger declares that she is employed by ISDIN. Bianca Maria Piraccini, Aurora Alessandrini, Nicolò Brandi, Francesca Bruni, Victor Desmond Mandel, Giovanni Pellacani, Michela Starace have nothing to disclose.

Compliance with Ethics Guidelines. Due to the cosmetic nature of the intended use of the product studied, ethics committee approval was not required. Both studies were performed in line with the Declaration of Helsinki (1964) and its subsequent amendments. In both studies, the subjects were informed about the expected benefits of the tested products and the constraints of study participation, and signed an informed consent form, including consent for the use of photographs taken during the studies, prior to the start of the studies.

Open Access. This article is distributed under the terms of the Creative Commons Attribution-NonCommercial 4.0 International License (http://creativecommons.org/licenses/ by-nc/4.0/), which permits any noncommercial use, distribution, and reproduction in any medium, provided you give appropriate credit to the original author(s) and the source, provide a link to the Creative Commons license, and indicate if changes were made. 


\section{REFERENCES}

1. Chessa MA, Lorizzo M, Richert B, Lopez Estebaranz JL, Rigopoulos D, Tosti A, Gupta AK, Di Chiacchio N, Di Chiacchio NG, Rubin A, Baran R, Lipner SR, Daniel R, Chiheb S, Grover C, Starace M, Piraccini BM. Brittle nails, clinical signs and treatment recommendations. Dermatol. Ther. 2019.

2. Lipner SR, Scher RK. Biotin for the treatment of nail disease: what is the evidence? J Dermatolog Treat. 2018;29(4):411-4.

3. Iorizzo M, Pazzaglia M, Piraccini B, Tullo S, Tosti A. Brittle nails. J Cosmet Dermatol. 2004;3(3):138-44.

4. Gequelim GC, Kubota CY, Sanches S, Dranka D, Mejia MM, Sumiya FM, Schmitt JV. Perception of brittle nails in dermatologic patients: a cross-sectional study. An Br Dermatol. 2013;88(6):1022-5.

5. Hexsel D, Zague V, Schunck M, Siega C, Camozzato FO, Oesser S. Oral supplementation with specific bioactive collagen peptides improves nail growth and reduces symptoms of brittle nails. J Cosmet Dermatol. 2017;16(4):520-6.

6. González S, Swindells K, Rajadhyaksha M, Torres A. Changing paradigms in dermatology: confocal microscopy in clinical and surgical dermatology. Clin Dermatol. 2003;21(5):359-69.

7. Cinotti E, Fouilloux B, Perrot JL, Labeille B, Douchet C, Cambazard F. Confocal microscopy for healthy and pathological nail. J Eur Acad Dermatol Venereol. 2014;28(7):853-8.

8. Trojahn C, Schario M, Dobos G, Blume-Peytavi U, Kottner J. Reliability and validity of two in vivo measurements for skin surface topography in aged adults. Skin Res Technol. 2015;21(1):54-60.

9. Berardesca E, Howard I, Wilhelm MP. Non-invasive diagnostic techniques in clinical dermatology. 2014. Chapter: PRIMOS 3D digital frame projection. Sören Jaspers, Christian Benderoth, pp. 41-54.

10. Ulrich M, Themstrup L, de Carvalho N, Manfredi M, Grana C, Ciardo S, Kästle R, Holmes J, Whitehead R, Jemec GB, Pellacani G, Welzel J. Dynamic optical coherence tomography in dermatology. Dermatology. 2016;232(3):298-311.

11. Manfredini M, Greco M, Farnetani F, Ciardo S, De Carvalho N, Mandel VD, et al. Acne: morphologic and vascular study of lesions and surrounding skin by means of optical coherence tomography. J Eur Acad Dermatol Venereol. 2017;31(9):1541-6.

12. Sattler E, Kaestle R, Rothmund G, Welzel J. Confocal laser scanning microscopy, optical coherence tomography and transonychial water loss for in vivo investigation of nails. $\mathrm{Br} \mathrm{J}$ Dermatol. 2012;166(4):740-6.

13. Manfredini M, Mazzaglia G, Ciardo S, Farnetani F, Mandel VD, Longo C, et al. Acne: in vivo morphologic study of lesions and surrounding skin by means of reflectance confocal microscopy. J Eur Acad Dermatol Venereol. 2015;29(5):933-9399.

14. Iorizzo M. Tips to treat the 5 most common nail disorders: brittle nails, onycholysis, paronychia, psoriasis, onychomycosis. Dermatol Clin. 2015;33(2):175-83.

15. Mertin D, Lippold BC. In-vitro permeability of the human nail and of a keratin membrane from bovine hooves: influence of the partition coefficient octanol/water and the water solubility of drugs on their permeability and maximum flux. J Pharm Pharmacol. 1997;49(1):30-4.

16. Uyttendaele H, Geyer A, Scher RK. Brittle nails: pathogenesis and treatment. J Drugs Dermatol. 2003;2(1):48-9.

17. Monti D, Saccomani L, Chetoni P, Burgalassi S, Tampucci S, Mailland F. Validation of bovine hoof slices as a model for infected human toenails: in vitro ciclopirox transungual permeation. $\mathrm{Br} \mathrm{J}$ Dermatol. 2011;165(1):99-105.

18. Piraccini BM, Alessandrini A, Starace M. Onychoscopy: dermoscopy of the nails. Dermatol Clin. 2018;36(4):431-8.

19. Aldahan AS, Chen LL, Fertig RM, Holmes J, Shah VV, Mlacker S, Hsu VM, Nouri K, Tosti A. Vascular features of nail psoriasis using dynamic optical coherence tomography. Skin Appendage Disord. 2017;2(3-4):102-8.

20. Aydin SZ, Castillo-Gallego C, Ash ZR, Abignano G, Marzo-Ortega $H$, Wittmann $M$, Del Galdo F, McGonagle D. Potential use of optical coherence tomography and high-frequency ultrasound for the assessment of nail disease in psoriasis and psoriatic arthritis. Dermatology. 2013;227:45-51.

21. Cinotti E, Labeille B, Cambazard F, Perrot JL. Confocal microscopy for special sites and special uses. Dermatol Clin. 2016;34(4):477-85. 